\begin{tabular}{l} 
O P E R A T I O N S R E S E A R C H A N D D E C I S I O N S \\
\hline No. 1
\end{tabular}

DOI: $10.37190 /$ ord200106

\title{
ASSESSMENT OF MIXED NETWORK PROCESSES WITH SHARED INPUTS AND UNDESIRABLE FACTORS
}

\author{
MARYAM NEMATIZADEH ${ }^{1}$, ALIREZA AMIRTEIMOORI ${ }^{1}$, \\ SOHRAB KORDROSTAMI $^{2 *}$, MOHSEN VAEZ-GHASEMI ${ }^{1}$ \\ ${ }^{1}$ Department of Applied Mathematics, Rasht Branch, Islamic Azad University, Rasht, Iran \\ ${ }^{2}$ Department of Mathematics, Lahijan Branch, Islamic Azad University, Lahijan, Iran
}

In the real world, there are processes whose structures are like a parallel-series mixed network. Network data envelopment analysis (NDEA) is one of the appropriate methods for assessing the performance of processes with these structures. In the paper, mixed processes with two parallel and series components are considered, in which the first component or parallel section consists of the shared inputs, and the second component or series section consists of undesirable factors. By considering the weak disposability assumption for undesirable factors, a DEA approach as based on network slackbased measure (NSBM) is introduced to evaluate the performance of processes with mixed structures. The proposed model is illustrated with a real case study. Then, the model is developed to discriminate efficient units.

Keywords: network data envelopment analysis, parallel-series mixed networks, weak disposability, undesirable factors, ranking

\section{Introduction}

Network data envelopment analysis (NDEA) is a non-parametric method to evaluate the relative efficiency of a set of homogeneous decision-making units (DMUs) with multiple inputs and outputs introduced by Färe and Grosskopf [8]. Contrary to conventional DEA models that neglect the internal structure of processes and only take into account external inputs and outputs to evaluate the performance of processes, all network models consider internal materials.

One of the basic network structures is the series two-stage process that has already been widely studied. We briefly review some of the studies carried out on these systems.

*Corresponding author S. Kordrostami, email address kordrostami@liau.ac.ir

Received 13 March 2019, accepted 16 April 2020 
Seiford and Zhu [18] apply the standard DEA model to evaluate the efficiency of each stage. Chen and Zhu [7] introduce a model under the assumption of variable returns to scale. In some cases, it may conclude that the inefficiency of two stages leads to overall efficiency. Kao and Hwang [11] modify the standard DEA model and develop the model with multiplicative function. Chen et al. [5] propose an additive model for evaluating the efficiency of two-stage processes. From the proposed models it can be concluded that the performance improvement in one stage leads to inefficiency of other stages. Liang et al. [13] introduce a new model and solve this problem. Tone and Tsutsui [21] propose a slack-based network DEA model to determine the relative efficiency score of network processes.

In the mentioned studies, there are not any undesirable factors in the network structures. However, in the structure of some network processes, there are undesirable factors and they play an important role in evaluating the performance of the processes. Thus, Maghbouli et al. [16] consider weak disposability assumption for undesirable factors and introduce a new model for assessing the efficiency of two-stage processes with undesirable outputs. Kao [10] develops a parallel model to evaluate the efficiency of systems with parallel structure. Zha and Liang [25] offer a cooperative efficiency model to evaluate the overall efficiency and the relation between the sub-sections of a two-stage network with shared inputs for both stages. Chen et al. [6] propose an approach for assessing the relative efficiency of two-stage processes with inseparable shared inputs. Amirteimoori et al. [1] introduce an additive model for evaluating the relative efficiency of two-stage processes with shared input resource in both stages. Also, Wu et al. [22] consider an additive efficiency model for parallel systems with shared inputs and undesirable outputs.

Moreover, there are systems with complex structures, as, e.g., mixtures of both parallel and series processes, in the real world and their performance should also be measured. In the DEA literature, some studies have addressed mixed structures. Yu and Lin [24] use a directional distance function to evaluate the efficiency of railways in which each line contains two parallel (production) and series (consumption) sections. Yu [23] uses the SBM model to examine the airport performance, divided into two sections of landside and airside. Based on the SBM model, Lin and Chiu [14] evaluate Taiwanese banks, the performance of which consisted of three parts of production, service and profitability. Moreover, Amirteimoori and Yang [2] introduce an additive model for assessing the efficiency of production processes with the two-stage parallel-series structure. Ma and Chen [15] consider a parallel-series structure and develop a hybrid DEA model for the mentioned structure. They apply their model to evaluate the Taiwanese non-life insurance companies.

In the above-mentioned studies, the structure of each process is different. Besides the number of components related to each structure and how they are put together, the presence of undesirable factors and also shared inputs makes them different from each other. Nevertheless, the evaluation complexity of these systems due to their structure 
and also addressing weakly disposable undesirable outputs makes a motivation for presenting a suitable model. The weak disposability assumption enables us to model undesirable outputs as outputs, taking into account the possible trade-offs between the desirable and undesirable outputs.

In this study, the purpose is to provide a DEA approach based on network slackbased measure (NSBM) model for assessing the performance of network processes with a mixed parallel-series structure, consisting shared inputs and undesirable factors. Also, we introduce a super-efficiency model for ranking efficient units. In the proposed models, we use weak disposability assumption for desirable and undesirable outputs. One of the advantages of the proposed approach is to calculate the overall efficiency of the whole system without the need to evaluate the efficiency of each of the subsections. Also, the optimal amount of the shared inputs of the mentioned system is determined.

The paper is organised as follows: the next section firstly introduces the proposed model to estimate the relative efficiency of systems with mixed network processes. Then, a super-efficiency model is introduced to discriminate efficient units. In Section 3, an application is presented to illustrate the proposed approach. Conclusions are given in Section 4.

\section{Proposed model for mixed processes}

We initially consider a mixed process as shown in Fig. 1 that consists of two parallel (A), and series (B) sections. Then, a novel model for evaluating the relative efficiency of the mentioned structure is introduced. After that, a super-efficiency model is presented to discriminate efficient units.

Now, suppose there are $K D M U$ s, and each $D M U_{k}, k=1, \ldots, K$ has the structure as can be seen in Fig. 1. Therefore, each $D M U_{k}, k=1, \ldots, K$ consists of three stages. The first and second stages comprise input vectors of $u_{k}^{(1)}=\left(u_{1 k}^{(1)}, \ldots, u_{R k}^{(1)}\right)$ and $u_{k}^{(2)}=\left(u_{1 k}^{(2)}, \ldots, u_{R k}^{(2)}\right)$, respectively. Also, both stages consume a shared input $x_{k}=\left(x_{1 k}, \ldots, x_{I k}\right)$ in which $x_{k}=x_{k}^{(1)}+x_{k}^{(2)}$, and the input vectors $x_{k}^{(1)}=\left(x_{1 k}^{(1)}, \ldots, x_{l k}^{(1)}\right)$ and $x_{k}^{(2)}=\left(x_{1 k}^{(2)}, \ldots, x_{l k}^{(2)}\right)$ are used for stages 1 and 2, respectively. Assume $\alpha^{(1)}+\alpha^{(2)}=1$. At optimality, the portion amount $0 \leq \alpha^{(1)}<1$ of the shared input to the first stage, and the rest portion $0 \leq \alpha^{(2)}<1$ of the shared input to the second stage are allocated. The first stage consumes inputs $u_{r k}^{(1)}, r=1, \ldots, R$ and $\alpha_{i}^{(1)} x_{i k}, i=1, \ldots, I$, and it produces the undesirable output $z_{k}^{(1)}=\left(z_{1 k}^{(1)}, \ldots, z_{T k}^{(1)}\right)$ and the final desirable output $v_{k}^{(1)}=\left(v_{1 k}^{(1)}, \ldots, v_{J k}^{(1)}\right)$. Moreover, the second stage uses inputs $u_{r k}^{(2)}, r=1, \ldots, R$ and $\alpha_{i}^{(2)} x_{i k}, i=1, \ldots, I$, and it produces the undesirable output $z_{k}^{(2)}=\left(z_{1 k}^{(2)}, \ldots, z_{T k}^{(2)}\right)$ and the final desirable output $v_{k}^{(2)}=\left(v_{1 k}^{(2)}, \ldots, v_{J k}^{(2)}\right)$. In addition to 
the usage of undesirable outputs $z_{k}^{(1)}, z_{k}^{(2)}$ as inputs, the third stage consumes the extra input $w_{k}=\left(w_{1 k}, \ldots, w_{M k}\right)$ to produce the desirable and undesirable outputs $h_{k}=\left(h_{1 k}, \ldots, h_{N k}\right)$ and $y_{k}=\left(y_{1 k}, \ldots, y_{L k}\right)$, respectively.

a)

b)

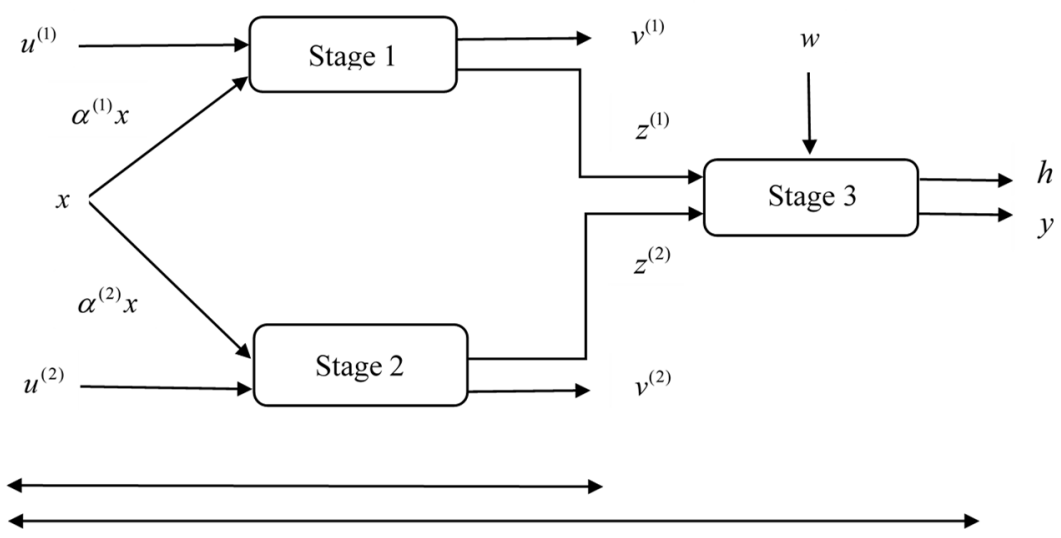

Fig. 1. A parallel-series mixed process

In 1970, Shephard [19] express desirable and undesirable outputs are weakly disposable if and only if their uniform reduction is feasible. As based on it, Färe and Grosskopf [9] use a single abatement factor for all units in Shephard's technology. Then, Kuosmanen [12] consider a distinctive contraction factor for each unit. Now, according to Fig. 1 and based on the technology proposed by Kuosmanen, the algebraic representation of the production possibility set under the variable returns to scale is defined as follows:

$$
T=\left\{\left(u^{(1)}, u^{(2)}, x^{(1)}, x^{(2)}, w, v^{(1)}, v^{(2)}, h, z^{(1)}, z^{(2)}, y\right)\right.
$$

Stage 1 constraints

$$
\begin{aligned}
& \sum_{k=1}^{K} \lambda_{k} u_{r k}^{(1)} \leq u_{r o}^{(1)}, r=1, \ldots, R \\
& \sum_{k=1}^{K} \lambda_{k} x_{i k}^{(1)} \leq \alpha_{i}^{(1)}\left(x_{i o}^{(1)}+x_{i o}^{(2)}\right), i=1, \ldots, I \\
& \sum_{k=1}^{K} \lambda_{k} \theta_{k} v_{j k}^{(1)} \geq v_{j o}^{(1)}, j=1, \ldots, J \\
& \sum_{k=1}^{K} \lambda_{k} \theta_{k} z_{t k}^{(1)}=z_{t o}^{(1)}, t=1, \ldots, T
\end{aligned}
$$


Stage 2 constraints

$$
\begin{aligned}
& \sum_{k=1}^{K} \lambda_{k} u_{r k}^{(2)} \leq u_{r o}^{(2)}, r=1, \ldots, R \\
& \sum_{k=1}^{K} \lambda_{k} x_{i k}^{(2)} \leq \alpha_{i}^{(2)}\left(x_{i o}^{(1)}+x_{i o}^{(2)}\right), i=1, \ldots, I \\
& \sum_{k=1}^{K} \lambda_{k} \theta_{k} v_{j k}^{(2)} \geq v_{j o}^{(2)}, j=1, \ldots, J \\
& \sum_{k=1}^{K} \lambda_{k} \theta_{k} z_{t k}^{(2)}=z_{t o}^{(2)}, t=1, \ldots, T
\end{aligned}
$$

Stage 3 constraints

$$
\begin{aligned}
& \sum_{k=1}^{K} \lambda_{k} w_{m k} \leq w_{m o}, m=1, \ldots, M \\
& \sum_{k=1}^{K} \lambda_{k} \theta_{k} z_{t k}^{(1)}=z_{t o}^{(1)}, t=1, \ldots, T \\
& \sum_{k=1}^{K} \lambda_{k} \theta_{k} z_{t k}^{(2)}=z_{t o}^{(2)}, t=1, \ldots, T \\
& \sum_{k=1}^{K} \lambda_{k} \theta_{k} h_{n k} \geq h_{n o}, n=1, \ldots, N \\
& \sum_{k=1}^{K} \lambda_{k} \theta_{k} y_{l k}=y_{l o}, l=1, \ldots, L
\end{aligned}
$$

Generic constraints:

$$
\begin{aligned}
& \sum_{k=1}^{K} \lambda_{k}=1 \\
& \alpha_{i}^{(1)}+\alpha_{i}^{(2)}=1, i=1, \ldots, I \\
& \left.\alpha_{i}^{(1)}, \alpha_{i}^{(2)} \geq 0 \forall i, \lambda_{k} \geq 0,0 \leq \theta_{k} \leq 1 \forall k\right\}
\end{aligned}
$$

The above technology consists of four parts. The first three sections present the constraints of each of the first, second and third stages, respectively. Also, the final section shows the general constraints of the system. $\theta_{k}, k=1, \ldots, K$, are unknown abatement factors which are introduced for undesirable and desirable outputs. Also, $\alpha^{(1)}$ and $\alpha^{(2)}$ are unknown portion amounts for shared inputs. The above technology is non-linear and, by following Kuosmanen [12], it can easily be transformed into the below linear technology: 


$$
T=\left\{\left(u^{(1)}, u^{(2)}, x^{(1)}, x^{(2)}, w, v^{(1)}, v^{(2)}, h, z^{(1)}, z^{(2)}, y\right)\right.
$$


Stage 1 constraints

$$
\begin{aligned}
& \sum_{k=1}^{K}\left(\rho_{k}+\mu_{k}\right) u_{r k}^{(1)} \leq u_{r o}^{(1)}, r=1, \ldots, R \\
& \sum_{k=1}^{K}\left(\rho_{k}+\mu_{k}\right) x_{i k}^{(1)} \leq \alpha_{i}^{(1)}\left(x_{i o}^{(1)}+x_{i o}^{(2)}\right), i=1, \ldots, I \\
& \sum_{k=1}^{K} \rho_{k} v_{j k}^{(1)} \geq v_{j o}^{(1)}, j=1, \ldots, J \\
& \sum_{k=1}^{K} \rho_{k} z_{t k}^{(1)}=z_{t o}^{(1)}, t=1, \ldots, T
\end{aligned}
$$

Stage 2 constraints

$$
\begin{aligned}
& \sum_{k=1}^{K}\left(\rho_{k}+\mu_{k}\right) u_{r k}^{(2)} \leq u_{r o}^{(2)}, r=1, \ldots, R \\
& \sum_{k=1}^{K}\left(\rho_{k}+\mu_{k}\right) x_{i k}^{(2)} \leq \alpha_{i}^{(2)}\left(x_{i o}^{(1)}+x_{i o}^{(2)}\right), i=1, \ldots, I \\
& \sum_{k=1}^{K} \rho_{k} v_{j k}^{(2)} \geq v_{j o}^{(2)}, j=1, \ldots, J \\
& \sum_{k=1}^{K} \rho_{k} z_{t k}^{(2)}=z_{t o}^{(2)}, t=1, \ldots, T
\end{aligned}
$$

Stage 3 constraints

$$
\begin{aligned}
& \sum_{k=1}^{K}\left(\rho_{k}+\mu_{k}\right) w_{m k} \leq w_{m o}, m=1, \ldots, M \\
& \sum_{k=1}^{K} \rho_{k} z_{t k}^{(1)}=z_{t o}^{(1)}, t=1, \ldots, T \\
& \sum_{k=1}^{K} \rho_{k} z_{t k}^{(2)}=z_{t o}^{(2)}, t=1, \ldots, T \\
& \sum_{k=1}^{K} \rho_{k} h_{n k} \geq h_{n o}, n=1, \ldots, N \\
& \sum_{k=1}^{K} \rho_{k} y_{l k}=y_{l o}, l=1, \ldots, L
\end{aligned}
$$

Generic constraints:

$$
\begin{aligned}
& \alpha_{i}^{(1)}+\alpha_{i}^{(2)}=1, i=1, \ldots, I \\
& \sum_{k=1}^{K}\left(\rho_{k}+\mu_{k}\right)=1 \\
& \left.\rho_{k}, \mu_{k}, \alpha_{i}^{(1)}, \alpha_{i}^{(2)} \geq 0 \forall k, i\right\}
\end{aligned}
$$


In linear technology (2), $\rho_{k}$ and $\mu_{k}$ are unknown variables in which $\rho_{k}=\theta_{k} \lambda_{k}$, $\mu_{k}=\left(1-\theta_{k}\right) \lambda_{k}$. We present the following fractional problem to achieve the efficiency score for the mixed process shown in Fig. 1:

$$
e_{o}^{*}=\min \frac{1-\frac{1}{R+I+M}\left[\sum_{r=1}^{R} \frac{s_{r o}^{\left(u^{(1)}\right)}+s_{r o}^{\left(u^{(2)}\right)}}{u_{r o}^{(1)}+u_{r o}^{(2)}}+\sum_{i=1}^{I} \frac{s_{i o}^{\left(x^{(1)}\right)}+s_{i o}^{\left(x^{(2)}\right)}}{x_{i o}^{(1)}+x_{i o}^{(2)}}+\sum_{m=1}^{M} \frac{s_{m o}^{(w)}}{w_{m o}}\right]}{1+\frac{1}{J+N}\left[\sum_{j=1}^{J} \frac{s_{j o}^{\left(v^{(1)}\right)}+s_{j o}^{\left(v^{(2)}\right)}}{v_{j o}^{(1)}+v_{j o}^{(2)}}+\sum_{n=1}^{N} \frac{s_{n o}^{(h)}}{h_{n o}}\right]}
$$

s.t.

Stage 1 constraints

$$
\begin{aligned}
& \sum_{k=1}^{K}\left(\rho_{k}+\mu_{k}\right) u_{r k}^{(1)}=u_{r o}^{(1)}-s_{r o}^{\left(u^{(1)}\right)}, r=1, \ldots, R \\
& \sum_{k=1}^{K}\left(\rho_{k}+\mu_{k}\right) x_{i k}^{(1)}=\alpha_{i}^{(1)}\left(x_{i o}^{(1)}+x_{i o}^{(2)}\right)-s_{i o}^{\left(x^{(1)}\right)}, i=1, \ldots, I \\
& \sum_{k=1}^{K} \rho_{k} v_{j k}^{(1)}=v_{j o}^{(1)}+s_{j o}^{\left(v^{(1)}\right)}, j=1, \ldots, J \\
& \sum_{k=1}^{K} \rho_{k} z_{t k}^{(1)}=z_{t o}^{(1)}, t=1, \ldots, T
\end{aligned}
$$

Stage 2 constraints

$$
\begin{aligned}
& \sum_{k=1}^{K}\left(\rho_{k}+\mu_{k}\right) u_{r k}^{(2)}=u_{r o}^{(2)}-s_{r o}^{\left(u^{(2)}\right)}, r=1, \ldots, R \\
& \sum_{k=1}^{K}\left(\rho_{k}+\mu_{k}\right) x_{i k}^{(2)}=\alpha_{i}^{(2)}\left(x_{i o}^{(1)}+x_{i o}^{(2)}\right)-s_{i o}^{\left(x^{(2)}\right)}, i=1, \ldots, I \\
& \sum_{k=1}^{K} \rho_{k} v_{j k}^{(2)}=v_{j o}^{(2)}+s_{j o}^{\left(v^{(2)}\right)}, j=1, \ldots, J \\
& \sum_{k=1}^{K} \rho_{k} z_{t k}^{(2)}=z_{t o}^{(2)}, t=1, \ldots, T
\end{aligned}
$$

Stage 3 constraints

$$
\begin{aligned}
& \sum_{k=1}^{K}\left(\rho_{k}+\mu_{k}\right) w_{m k}=w_{m o}-s_{m o}^{(w)}, m=1, \ldots, M \\
& \sum_{k=1}^{K} \rho_{k} z_{t k}^{(1)}=z_{t o}^{(1)}, t=1, \ldots, T \\
& \sum_{k=1}^{K} \rho_{k} z_{t k}^{(2)}=z_{t o}^{(2)}, t=1, \ldots, T
\end{aligned}
$$




$$
\begin{aligned}
& \sum_{k=1}^{K} \rho_{k} h_{n k}=h_{n o}+s_{n o}^{(h)}, n=1, \ldots, N \\
& \sum_{k=1}^{K} \rho_{k} y_{l k}=y_{l o}, l=1, \ldots, L
\end{aligned}
$$

Generic constraints

$$
\begin{aligned}
& \alpha_{i}^{(1)}+\alpha_{i}^{(2)}=1, i=1, \ldots, I \\
& \sum_{k=1}^{K}\left(\rho_{k}+\mu_{k}\right)=1 \\
& \alpha_{i}^{(1)}, \alpha_{i}^{(2)}, \rho_{k}, \mu_{k} \geq 0 \forall i, k \\
& s_{r o}^{\left(u^{(1)}\right)}, s_{r o}^{\left(u^{(2)}\right)}, s_{i o}^{\left(x^{(1)}\right)}, s_{i o}^{\left(x^{(2)}\right)}, s_{j o}^{\left(v^{(1)}\right)}, s_{j o}^{\left(v^{(2)}\right)}, s_{m o}^{(w)}, s_{n o}^{(h)} \geq 0, \forall r, i, j, m, n
\end{aligned}
$$

The above model is constructed on the slacks-based measure model. $s_{r o}^{\left(u^{(1)}\right)}, s_{r o}^{\left(u^{(2)}\right)}$, $s_{i o}^{\left(x^{(1)}\right)}, s_{i o}^{\left(x^{(2)}\right)}, s_{j o}^{\left(v^{(1)}\right)}, s_{j o}^{\left(v^{(2)}\right)}, s_{m o}^{(w)}, s_{n o}^{(h)}$ are unknown slack variables and positive for the constraints of inputs and desirable outputs. Note that model (3) is non-linear, and it can be converted to the linear problem using the Charnes-Cooper transformation [4].

Let

$$
\frac{1}{1+\frac{1}{J+N}\left[\sum_{j=1}^{J} \frac{s_{j o}^{\left(v^{(1)}\right)}+s_{j o}^{\left(v^{(2)}\right)}}{v_{j o}^{(1)}+v_{j o}^{(2)}}+\sum_{n=1}^{N} \frac{s_{n o}^{(h)}}{h_{n o}}\right]}=\delta(>0), \delta \alpha_{i}=\beta_{i}, \delta \rho_{k}=\gamma_{k},
$$

$\delta \mu_{k}=\eta_{k}, \delta s_{r}=q_{r}, \delta s_{i}=q_{i}, \delta s_{j}=q_{j}, \delta s_{m}=q_{m}, \delta s_{n}=q_{n}$ and $\delta s_{l}=q_{l}$. Then, model (3) transformed into the linear program is as follows:

$$
\mathrm{e}_{o}^{*}=\min \left[\delta-\frac{1}{R+I+M}\left(\sum_{r=1}^{R} \frac{q_{r o}^{\left(u^{(1)}\right)}+q_{r o}^{\left(u^{(2)}\right)}}{u_{r o}^{(1)}+u_{r o}^{(2)}}+\sum_{i=1}^{I} \frac{q_{i o}^{\left(x^{(1)}\right)}+q_{i o}^{\left(x^{(2)}\right)}}{x_{i o}^{(1)}+x_{i o}^{(2)}}+\sum_{m=1}^{M} \frac{q_{m o}^{(w)}}{w_{m o}}\right)\right]
$$

s.t.

Stage 1 constraints

$$
\begin{aligned}
& \sum_{k=1}^{K}\left(\gamma_{k}+\eta_{k}\right) u_{r k}^{(1)}=\delta u_{r o}^{(1)}-q_{r o}^{\left(u^{(1)}\right)}, r=1, \ldots, R \\
& \sum_{k=1}^{K}\left(\gamma_{k}+\eta_{k}\right) x_{i k}^{(1)}=\beta_{i}^{(1)}\left(x_{i o}^{(1)}+x_{i o}^{(2)}\right)-q_{i o}^{\left(x^{(1)}\right)}, i=1, \ldots, I \\
& \sum_{k=1}^{K} \gamma_{k} v_{j k}^{(1)}=\delta v_{j o}^{(1)}+q_{j o}^{\left(v^{(1)}\right)}, j=1, \ldots, J \\
& \sum_{k=1}^{K} \gamma_{k} z_{t k}^{(1)}=\delta z_{t o}^{(1)}, t=1, \ldots, T
\end{aligned}
$$


Stage 2 constraints

$$
\begin{aligned}
& \sum_{k=1}^{K}\left(\gamma_{k}+\eta_{k}\right) u_{r k}^{(2)}=\delta u_{r o}^{(2)}-q_{r o}^{\left(u^{(2)}\right)}, r=1, \ldots, R \\
& \sum_{k=1}^{K}\left(\gamma_{k}+\eta_{k}\right) x_{i k}^{(2)}=\beta_{i}^{(2)}\left(x_{i o}^{(1)}+x_{i o}^{(2)}\right)-q_{i o}^{\left(x^{(2)}\right)}, i=1, \ldots, I \\
& \sum_{k=1}^{K} \gamma_{k} v_{j k}^{(2)}=\delta v_{j o}^{(2)}+q_{j o}^{\left(v^{(2)}\right)}, j=1, \ldots, J \\
& \sum_{k=1}^{K} \gamma_{k} z_{t k}^{(2)}=\delta z_{t o}^{(2)}, t=1, \ldots, T
\end{aligned}
$$

Stage 3 constraints

$$
\begin{aligned}
& \sum_{k=1}^{K}\left(\gamma_{k}+\eta_{k}\right) w_{m k}=\delta w_{m o}-q_{m o}^{(w)}, m=1, \ldots, M \\
& \sum_{k=1}^{K} \gamma_{k} z_{t k}^{(1)}=\delta z_{t o}^{(1)}, t=1, \ldots, T \\
& \sum_{k=1}^{K} \gamma_{k} z_{t k}^{(2)}=\delta z_{t o}^{(2)}, t=1, \ldots, T \\
& \sum_{k=1}^{K} \gamma_{k} h_{n k}=\delta h_{n o}+q_{n o}^{(h)}, n=1, \ldots, N \\
& \sum_{k=1}^{K} \gamma_{k} y_{l k}=\delta y_{l o}, l=1, \ldots, L
\end{aligned}
$$

Generic constraints

$$
\begin{aligned}
& \delta+\frac{1}{J+N}\left[\sum_{j=1}^{J} \frac{q_{j o}^{(v)}+q_{j o}^{(v)}}{v_{j o}^{(1)}+v_{j o}^{(2)}}+\sum_{n=1}^{N} \frac{q_{n o}^{(h)}}{h_{n o}}\right]=1 \\
& \beta_{i}^{(1)}+\beta_{i}^{(2)}=\delta, i=1, \ldots, I \\
& \sum_{k=1}^{K}\left(\gamma_{k}+\eta_{k}\right)=\delta \\
& \delta>0, \gamma_{k}, \eta_{k}, \beta_{i}^{(1)}, \beta_{i}^{(2)} \geq 0 \forall k, i \\
& q_{r}^{\left(u^{(1)}\right)}, q_{i}^{\left(x^{(1)}\right)}, q_{j}^{\left(v^{(1)}\right)}, q_{r}^{\left(u^{(2)}\right)}, q_{i}^{\left(x^{(2)}\right)}, q_{j}^{\left(v^{(2)}\right)}, q_{m}^{(w)}, q_{n}^{(h)} \geq 0 \forall r, i, j, m, n
\end{aligned}
$$

It can be seen that model (4) is linear and feasible. Also, it consists of three groups of constraints related to the subsections of the mixed process. Minimum achievable efficiency, which is denoted by $e_{o}^{*}$, is the whole system's overall efficiency. Moreover, 
by solving model (4), the optimal portion values $\alpha^{(1)}$ and $\alpha^{(2)}$ are obtained. These portions show which stage and how much shared input is needed.

Theorem 1. The linear model (4) is feasible.

Proof. Consider

$$
\begin{aligned}
& \gamma_{o}=1, \eta_{k}=0, k=1, \ldots, K, k \neq 0 \\
& q^{\left(u^{(1)}\right)}=q^{\left(x^{(1)}\right)}=q^{\left(v^{(1)}\right)}=q^{\left(u^{(2)}\right)}=q^{\left(x^{(2)}\right)}=q^{\left(v^{(2)}\right)}=q^{(w)}=q^{(h)}=0 \\
& \delta=1, \beta_{i}^{(1)}=\frac{x_{i o}^{(1)}}{x_{i o}^{(1)}+x_{i o}^{(2)}}, \beta_{i}^{(2)}=\frac{x_{i o}^{(2)}}{x_{i o}^{(1)}+x_{i o}^{(2)}}, i=1, \ldots, I
\end{aligned}
$$

It is a feasible solution to this problem.

Definition 1. The unit under evaluation $D M U_{o}$ is overall efficient if and only if $e_{o}^{*}=1$. Also, if all slack variables are zero, then $D M U_{o}$ is a Pareto efficient. By solving model (4), we can obtain the optimal efficiency score for stages 1, 2 and 3 as follows:

$$
\begin{gathered}
e_{o}^{* \text { Stage } 1}=\frac{1-\frac{1}{R+I}\left[\sum_{r=1}^{R} \frac{s_{r o}^{\left(u^{(1)}\right)^{*}}}{u_{r o}^{(1)}}+\sum_{i=1}^{I} \frac{s_{i o}^{\left(x^{(1)} *^{*}\right.}}{x_{i o}^{(1)}}\right]}{1+\frac{1}{J} \sum_{j=1}^{J} \frac{s_{j o}^{\left(\left(^{(1)}\right)^{*}\right.}}{v_{j o}^{(1)}}} \\
e_{o}^{* \text { Stage 2 }}=\frac{1-\frac{1}{R+I}\left[\sum_{r=1}^{R} \frac{s_{r o}^{\left(u^{(2)}\right)^{*}}}{u_{r o}^{(2)}}+\sum_{i=1}^{I} \frac{s_{i o}^{\left(x^{(2)}\right)^{*}}}{x_{i o}^{(2)}}\right]}{1+\frac{1}{J} \sum_{j=1}^{J} \frac{s_{j o}^{\left(v^{(2)}\right)^{*}}}{v_{j o}^{(2)}}} \\
e_{o}^{* \text { Stage 3 }}=\frac{1-\frac{1}{M} \sum_{m=1}^{M} \frac{s_{m o}^{(w)^{*}}}{w_{m o}}}{1+\frac{1}{N} \sum_{n=1}^{N} \frac{s_{n o}^{(h)^{*}}}{h_{n o}}}
\end{gathered}
$$

where $s_{r o}^{\left(u^{(1)}\right)^{*}}, s_{r o}^{\left(u^{(2)}\right)^{*}}, s_{i o}^{\left(x^{(1)}\right)^{*}}, s_{i o}^{\left(x^{(2)}\right)^{*}}, s_{j o}^{\left(v^{(1)}\right)^{*}}, s_{j o}^{\left(v^{(2)}\right)^{*}}, s_{m o}^{(w)^{*}}, s_{n o}^{(h)^{*}}$ are the optimal values of slack variables obtained from model (4). Since $\delta^{*}$ is positive, we can obtain an optimal solution 
to model (3) from $s^{\left(u^{(1)}\right)^{*}}=\frac{q^{\left(u^{(1)}\right)^{*}}}{\delta^{*}}, s^{\left(u^{(2)}\right)^{*}}=\frac{q^{\left(u^{(2)}\right)^{*}}}{\delta^{*}}, s^{\left(x^{(1)}\right)^{*}}=\frac{q^{\left(x^{(1)}\right)^{*}}}{\delta^{*}}, s^{\left(x^{(2)}\right)^{*}}=\frac{q^{\left(x^{(2)}\right)^{*}}}{\delta^{*}}$, $s^{\left(v^{(1)}\right)^{*}}=\frac{q^{\left(v^{(1)}\right)^{*}}}{\delta^{*}}, s^{\left(v^{(2)}\right)^{*}}=\frac{q^{\left(v^{(2)}\right)^{*}}}{\delta^{*}}, s^{(w)^{*}}=\frac{q^{(w)^{*}}}{\delta^{*}}, s^{(h)^{*}}=\frac{q^{(h)^{*}}}{\delta^{*}}$.

Definition 2. Components 1, 2 and 3 of the unit under evaluation $D M U_{o}$ are called efficient if and only if $e_{o}^{* \text { Stage } 1}=1, e_{o}^{* \text { Stage } 2}=1$, and $e_{o}^{* \text { Stage } 3}=1$.

By solving model (4), some units are determined as efficient, while others become inefficient. To discriminate among the efficient units, we present a ranking method based on the super-efficiency model, introduced by Tone [20], for the mentioned process as follows:

$$
\begin{aligned}
\sigma_{o}^{*}= & \min \frac{\frac{1}{R+I+M+T+L}}{\frac{1}{J+N}} \\
& \times \frac{\left(\sum_{r=1}^{R} \frac{\bar{u}_{r k}^{(1)}+\bar{u}_{r k}^{(2)}}{u_{r o}^{(1)}+u_{r o}^{(2)}}+\sum_{i=1}^{I} \frac{\bar{x}_{i k}^{(1)}+\bar{x}_{i k}^{(2)}}{x_{i o}^{(1)}+x_{i o}^{(2)}}+\sum_{m=1}^{M} \frac{\bar{w}_{m k}}{w_{m o}}+\sum_{t=1}^{T} \frac{\bar{z}_{t k}^{(1)}+\bar{z}_{t k}^{(2)}}{z_{t o}^{(1)}+z_{t o}^{(2)}}+\sum_{l=1}^{L} \frac{\bar{y}_{l k}}{y_{l o}}\right)}{\left(\sum_{j=1}^{J} \frac{\bar{v}_{j k}^{(1)}+\bar{v}_{j k}^{(2)}}{v_{j o}^{(1)}+v_{j o}^{(2)}}+\sum_{n=1}^{N} \frac{\bar{h}_{n k}}{h_{n o}}\right)}
\end{aligned}
$$

s.t.

Stage 1 constraints

$$
\begin{aligned}
& \sum_{k=1, k \neq o}^{K}\left(\rho_{k}+\mu_{k}\right) u_{r k}^{(1)} \leq \bar{u}_{r k}^{(1)}, r=1, \ldots, R \\
& \sum_{k=1, k \neq o}^{K}\left(\rho_{k}+\mu_{k}\right) x_{i k}^{(1)} \leq \bar{x}_{i k}^{(1)}, i=1, \ldots, I \\
& \sum_{k=1, k \neq o}^{K} \rho_{k} v_{j k}^{(1)} \geq \bar{v}_{j}^{(1)}, j=1, \ldots, J \\
& \sum_{k=1, k \neq o}^{K} \rho_{k} z_{t k}^{(1)}=\bar{z}_{t k}^{(1)}, t=1, \ldots, T
\end{aligned}
$$

Stage 2 constraints

$$
\begin{aligned}
& \sum_{k=1, k \neq o}^{K}\left(\rho_{k}+\mu_{k}\right) u_{r k}^{(2)} \leq \bar{u}_{r k}^{(2)}, r=1, \ldots, R \\
& \sum_{k=1, k \neq o}^{K}\left(\rho_{k}+\mu_{k}\right) x_{i k}^{(2)} \leq \bar{x}_{i k}^{(2)}, i=1, \ldots, I
\end{aligned}
$$




$$
\begin{gathered}
\sum_{k=1, k \neq o}^{K} \rho_{k} v_{j k}^{(2)} \geq \bar{v}_{j k}^{(2)}, j=1, \ldots, J \\
\sum_{k=1, k \neq o}^{K} \rho_{k} z_{t k}^{(2)}=\bar{z}_{t k}^{(2)}, t=1, \ldots, T
\end{gathered}
$$

Stage 3 constraints

$$
\begin{aligned}
& \sum_{k=1, k \neq o}^{K}\left(\rho_{k}+\mu_{k}\right) w_{m k} \leq \bar{w}_{m k}, m=1, \ldots, M \\
& \sum_{k=1, k \neq o}^{K} \rho_{k} z_{t k}^{(1)}=\bar{z}_{t k}^{(1)}, t=1, \ldots, T \\
& \sum_{k=1, k \neq o}^{K} \rho_{k} z_{t k}^{(2)}=\bar{z}_{t k}^{(2)}, t=1, \ldots, T \\
& \sum_{k=1, k \neq o}^{K} \rho_{k} h_{n k} \geq \bar{h}_{n k}, n=1, \ldots, N \\
& \sum_{k=1, k \neq o}^{K} \rho_{k} y_{l k}=\bar{y}_{l k}, l=1, \ldots, L
\end{aligned}
$$

Generic constraints

$$
\begin{aligned}
& \alpha_{i}^{(1)}+\alpha_{i}^{(2)}=1, i=1, \ldots, I \\
& \sum_{k=1, k \neq o}^{K}\left(\rho_{k}+\mu_{k}\right)=1 \\
& \bar{u}_{r k}^{(1)} \geq u_{r o}^{(1)}, r=1, \ldots, R \\
& \bar{u}_{r k}^{(2)} \geq u_{r o}^{(2)}, r=1, \ldots, R \\
& \bar{x}_{i k}^{(1)} \geq \alpha_{i}^{(1)}\left(x_{i o}^{(1)}+x_{i o}^{(2)}\right), i=1, \ldots, I \\
& \bar{x}_{i k}^{(2)} \geq \alpha_{i}^{(2)}\left(x_{i o}^{(1)}+x_{i o}^{(2)}\right), i=1, \ldots, I \\
& \bar{w}_{m k} \geq w_{m o}, m=1, \ldots, M \\
& 0 \leq \bar{v}_{j k}^{(1)} \leq v_{j o}^{(1)}, j=1, \ldots, J \\
& 0 \leq \bar{v}_{j k}^{(2)} \leq v_{j o}^{(2)}, j=1, \ldots, J \\
& 0 \leq \bar{h}_{n k} \leq h_{n o}, n=1, \ldots, N \\
& \bar{z}_{t k}^{(1)} \geq 0, t=1, \ldots, T \\
& \bar{z}_{t k}^{(2)} \geq 0, t=1, \ldots, T \\
& \bar{y}_{l k} \geq 0, l=1, \ldots, L \\
& \rho_{k}, \mu_{k}, \alpha_{i}^{(1)}, \alpha_{i}^{(2)} \geq 0 \forall k, i
\end{aligned}
$$


In model (8), $\bar{u}_{r k}^{(1)}, \bar{u}_{r k}^{(2)}, \bar{x}_{i k}^{(1)}, \bar{x}_{i k}^{(2)}, \bar{z}_{t k}^{(1)}, \bar{z}_{t k}^{(2)}, \bar{v}_{j k}^{(1)}, \bar{v}_{j k}^{(2)}, \bar{h}_{n k}, \bar{w}_{m k}, \bar{y}_{l k}$ are unknown variables. Indeed, they are the maximum and minimum scores for the inputs and outputs obtained through the above model to calculate the super-efficiency for the efficient units. All of these are introduced in the generic constraints section above. The objective function is fractional, and it should be noted that the above model is nonlinear. Thus, considering

$$
\varphi=\frac{1}{\frac{1}{J+N}\left(\sum_{j=1}^{J} \frac{\bar{v}_{j k}^{(1)}+\bar{v}_{j k}^{(2)}}{v_{j o}^{(1)}+v_{j o}^{(2)}}+\sum_{n=1}^{N} \frac{\bar{h}_{n k}}{h_{n o}}\right)}
$$

for which $\varphi>0$, and also $\varphi \alpha_{i}=\beta_{i}, \varphi \rho_{k}=\gamma_{k}, \varphi \mu_{k}=\eta_{k}, \varphi \bar{x}_{i k}^{(1)}=\hat{x}_{i k}^{(1)}, \varphi \bar{x}_{i k}^{(2)}=\hat{x}_{i k}^{(2)}$, $\varphi \bar{u}_{r k}^{(1)}=\hat{u}_{r k}^{(1)}, \varphi \bar{u}_{r k}^{(2)}=\hat{u}_{r k}^{(2)}, \varphi \bar{v}_{j k}^{(1)}=\hat{v}_{j k}^{(1)}, \varphi \bar{v}_{j k}^{(2)}=\hat{v}_{j k}^{(2)}, \varphi \bar{z}_{t k}^{(1)}=\hat{z}_{t k}^{(1)}, \varphi \bar{z}_{t k}^{(2)}=\hat{z}_{t k}^{(2)}, \varphi \bar{w}_{m k}=\hat{w}_{m k}$, $\varphi \bar{h}_{n k}=\hat{h}_{n k}, \varphi \bar{y}_{l k}=\hat{y}_{l k}$, model (8) can be transformed into the following linear model:

$$
\begin{aligned}
\sigma_{o}^{*}= & \min \left[\frac { 1 } { R + I + M + T + L } \left(\sum_{r=1}^{R} \frac{\hat{u}_{r k}^{(1)}+\hat{u}_{r k}^{(2)}}{u_{r o}^{(1)}+u_{r o}^{(2)}}+\sum_{i=1}^{I} \frac{\hat{x}_{i k}^{(1)}+\hat{x}_{i k}^{(2)}}{x_{i o}^{(1)}+x_{i o}^{(2)}}\right.\right. \\
& \left.\left.+\sum_{m=1}^{M} \frac{\hat{w}_{m k}}{w_{m o}}+\sum_{t=1}^{T} \frac{\hat{z}_{t k}^{(1)}+\hat{z}_{t k}^{(2)}}{z_{t o}^{(1)}+z_{t o}^{(2)}}+\sum_{l=1}^{L} \frac{\hat{y}_{l k}}{y_{l o}}\right)\right]
\end{aligned}
$$

s.t.

Stage 1 constraints

$$
\begin{aligned}
& \sum_{k=1, k \neq o}^{K}\left(\gamma_{k}+\eta_{k}\right) u_{r k}^{(1)} \leq \hat{u}_{r k}^{(1)}, r=1, \ldots, R \\
& \sum_{k=1, k \neq o}^{K}\left(\gamma_{k}+\eta_{k}\right) x_{i k}^{(1)} \leq \hat{x}_{i k}^{(1)}, i=1, \ldots, I \\
& \sum_{k=1, k \neq o}^{K} \gamma_{k} v_{j k}^{(1)} \geq \hat{v}_{j}^{(1)}, j=1, \ldots, J \\
& \sum_{k=1, k \neq o}^{K} \gamma_{k} z_{t k}^{(1)}=\hat{z}_{t k}^{(1)}, t=1, \ldots, T
\end{aligned}
$$

Stage 2 constraints

$$
\sum_{k=1, k \neq o}^{K}\left(\gamma_{k}+\eta_{k}\right) u_{r k}^{(2)} \leq \hat{u}_{r k}^{(2)}, r=1, \ldots, R
$$




$$
\begin{aligned}
& \sum_{k=1, k \neq o}^{K}\left(\gamma_{k}+\eta_{k}\right) x_{i k}^{(2)} \leq \hat{x}_{i k}^{(2)}, i=1, \ldots, I \\
& \sum_{k=1, k \neq o}^{K} \gamma_{k} v_{j k}^{(2)} \geq \hat{v}_{j k}^{(2)}, j=1, \ldots, J \\
& \sum_{k=1, k \neq o}^{K} \gamma_{k} z_{t k}^{(2)}=\hat{z}_{t k}^{(2)}, t=1, \ldots, T
\end{aligned}
$$

Stage 3 constraints

$$
\begin{aligned}
& \sum_{k=1, k \neq 0}^{K}\left(\gamma_{k}+\eta_{k}\right) w_{m k} \leq \hat{w}_{m k}, m=1, \ldots, M \\
& \sum_{k=1, k \neq 0}^{K} \gamma_{k} z_{t k}^{(1)}=\hat{z}_{t k}^{(1)}, t=1, \ldots, T \\
& \sum_{k=1, k \neq 0}^{K} \gamma_{k} z_{t k}^{(2)}=\hat{z}_{t k}^{(2)}, t=1, \ldots, T \\
& \sum_{k=1, k \neq 0}^{K} \gamma_{k} h_{n k} \geq \hat{h}_{n k}, n=1, \ldots, N \\
& \sum_{k=1, k \neq o}^{K} \gamma_{k} y_{l k}=\hat{y}_{l k}, l=1, \ldots, L
\end{aligned}
$$

\section{Generic constraints}

$$
\begin{aligned}
& \frac{1}{J+N}\left(\sum_{j=1}^{J} \frac{\hat{v}_{j k}^{(1)}+\hat{v}_{j k}^{(2)}}{v_{j o}^{(1)}+v_{j o}^{(2)}}+\sum_{n=1}^{N} \frac{\hat{h}_{n k}}{h_{n o}}\right)=1 \\
& \beta_{i}^{(1)}+\beta_{i}^{(2)}=\varphi, i=1, \ldots, I \\
& \sum_{k=1, k \neq o}^{K}\left(\gamma_{k}+\eta_{k}\right)=\varphi \\
& \hat{u}_{r k}^{(1)} \geq \varphi u_{r o}^{(1)}, r=1, \ldots, R \\
& \hat{u}_{r k}^{(2)} \geq \varphi u_{r o}^{(2)}, r=1, \ldots, R \\
& \hat{x}_{i k}^{(1)} \geq \beta_{i}^{(1)}\left(x_{i o}^{(1)}+x_{i o}^{(2)}\right), i=1, \ldots, I \\
& \hat{x}_{i k}^{(2)} \geq \beta_{i}^{(2)}\left(x_{i o}^{(1)}+x_{i o}^{(2)}\right), i=1, \ldots, I \\
& \hat{w}_{m k} \geq \varphi w_{m o}, m=1, \ldots, M
\end{aligned}
$$




$$
\begin{aligned}
& 0 \leq \hat{v}_{j k}^{(1)} \leq \varphi v_{j o}^{(1)}, j=1, \ldots, J \\
& 0 \leq \hat{v}_{j k}^{(2)} \leq \varphi v_{j o}^{(2)}, j=1, \ldots, J \\
& 0 \leq \hat{h}_{n k} \leq \varphi h_{n o}, n=1, \ldots, N \\
& \hat{z}_{t k}^{(1)} \geq 0, t=1, \ldots, T \\
& \hat{z}_{t k}^{(2)} \geq 0, t=1, \ldots, T \\
& \hat{y}_{l k} \geq 0, l=1, \ldots, L \\
& \varphi>0, \gamma_{k}, \eta_{k}, \beta_{i}^{(1)}, \beta_{i}^{(2)} \geq 0, \forall k, i
\end{aligned}
$$

The above super-efficiency model is managed by removing the efficient unit under evaluation and calculating its distance to the new efficiency frontier. Any efficient unit that has the greatest distance gets a better ranking among the efficient units.

\section{Application}

Nowadays, one of the most important problems that many countries encounter is the water shortage. For this reason, many countries have sought to provide water for life through a variety of methods. One of the countries that have taken actions in this regard is China. In this regard, some researchers [3, 26, 17] have done studies in the evaluation of the WUWT systems. The WUWT systems consist of two sections, namely, water use (WU) and wastewater treatment (WT). In the mentioned structure, there are undesirable factors, and also in some, there are feedback outputs. The aforementioned studies considered the WUWT systems as two-stage structures.

Now, we consider the WUWT systems as a mixed structure. Then, as based on the models proposed in the previous section, we evaluate the efficiency of the WUWT systems. Therefore, we consider the WUWT systems of 31 regions in China that consist of two parallel and series sections that they have a mixed network structure (Fig. 2). This system has shared inputs and undesirable factors. The parallel section or WU section of each area contains 2 sectors. These sectors are divided into four categories: industry, agriculture, household, and environment. Industrial and agricultural categories use water as input for manufacturing, cooling, cleaning, farms, animals, forestry, and so on. Also, they use another input such as capital that is shared input. Household and environmental categories use water as input for urban and rural areas and rivers, lakes, and so on. Besides, they use shared input, namely, the capital. 


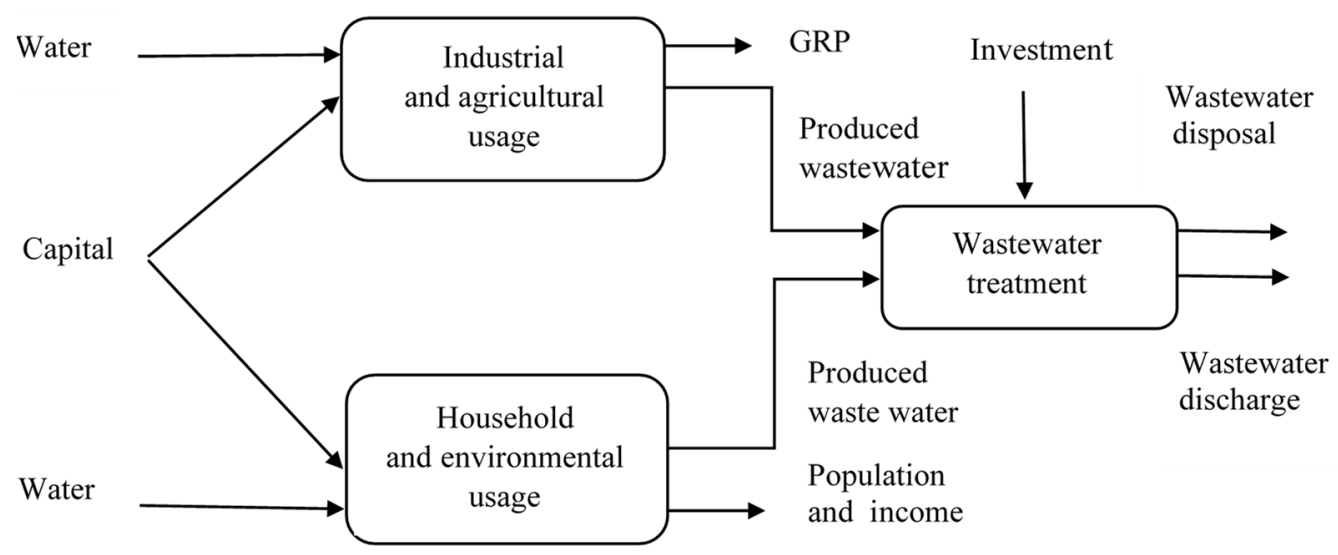

Fig. 2. Treated wastewater

The four categories produce wastewater as an undesirable output. Also, the industrial and agricultural usage sector produces GRP as a final desirable output. Moreover, the household and environmental usage component produce population and income as final desirable outputs. Undoubtedly, water is one of the important life factors. Hence, the existence of water in every place contributes to population growth. Increasing population leads to economic growth, rising productions level, taking taxes, water-rate, gasrate and items like that, all of which increase incomes of those areas. The third section or wastewater treatment (WT) consumes wastewater and investment as inputs and produces two final desirable and undesirable outputs, namely, wastewater disposal and wastewater discharge. The inputs and outputs of each mixed system are as follows:

- Industrial and agricultural usage

Inputs

- water needed for industrial and agricultural usage $\left(u^{(1)}\right)$,

- capital for water supply $\left(x^{(1)}\right)$.

Desirable output

- gross regional production score obtained from the industrial and agricultural part $\left(v^{(1)}\right)$.

Undesirable output

- wastewater discharged from industrial and agricultural products $\left(z^{(1)}\right)$.

- Household and environmental usage

Inputs

- water needed for household and environmental consumptions $\left(u^{(2)}\right)$,

- capital for water supply costs $\left(x^{(2)}\right)$.

Desirable outputs

- urban population proportion $\left(v_{1}^{(2)}\right)$,

- income of urban household disposable per capita $\left(v_{2}^{(2)}\right)$. 
Undesirable output

- wastewater discharged from the household and environmental products $\left(z^{(2)}\right)$.

- Wastewater treatment

Inputs

- this part, in addition to the usage of undesirable outputs of both parts 1 and $2,\left(z^{(1)}, z^{(2)}\right)$ as inputs, has another input, namely the investment to treat wastewater from the previous parts $(w)$.

Desirable output

- value of the wastewater treatment from the wastewater produced by the previous parts, namely $(h)$.

Undesirable output

- amount of wastewater not recoverable from wastewater from the previous parts, namely $(y)$.

In what follows, the statistical summary of the inputs consumed and the outputs produced by each of the three parts from 31 regions in China is given in Table 1. Data related to the year 2017 assembled from China Statistical Yearbook (National Bureau of Statistics of China).

Table 1. Statistical summary of data

\begin{tabular}{|c|c|c|c|c|}
\hline Variable & Mean & Minimum & Maximum & Standard deviation \\
\hline$u^{(1)}$ & 164.1806 & 9.8 & 545 & 132.4493 \\
\hline$u^{(2)}$ & 31.4258 & 2.8 & 105.3 & 20.6891 \\
\hline$x^{(1)}$ & 952.6290 & 111.8 & 2499.6 & 591.7728 \\
\hline$x^{(2)}$ & 2210.8129 & 193.5 & 4526.2 & 1345.008 \\
\hline$v_{1}^{(1)}$ & 8945.5806 & 198 & 36599 & 8826.8971 \\
\hline$v_{1}^{(2)}$ & 57039.9354 & 3042 & 183780 & 49199.4559 \\
\hline$v_{2}^{(2)}$ & 25163.7193 & 1151.4 & 80854.9 & 19776.0527 \\
\hline$z^{(1)}$ & 32290.7645 & 25693.5 & 57691.7 & 8126.3369 \\
\hline$z^{(2)}$ & 57.8477 & 29.56 & 87.9 & 12.2540 \\
\hline$w$ & 34916 & 15 & 158518 & 37484.4177 \\
\hline$h$ & 229385.5806 & 6143 & 938261 & 191067.3714 \\
\hline$y$ & 634.6387 & 42 & 2300.6 & 487.5307 \\
\hline
\end{tabular}

The performance of each area is evaluated by models (4) and (9) introduced in the previous section, and the results obtained are depicted in Fig. 3. Also, the details of the efficiencies of the whole system and their subsections are given in Table 2.

Lines in Fig. 3 show the overall and stages efficiency scores of 31 regions. Some lines are tangent and other are not. The tangent lines show which regions are overall efficient and the non-tangent lines indicate which regions are not total efficient. The efficiency values for these regions are also provided in Table 2 . 


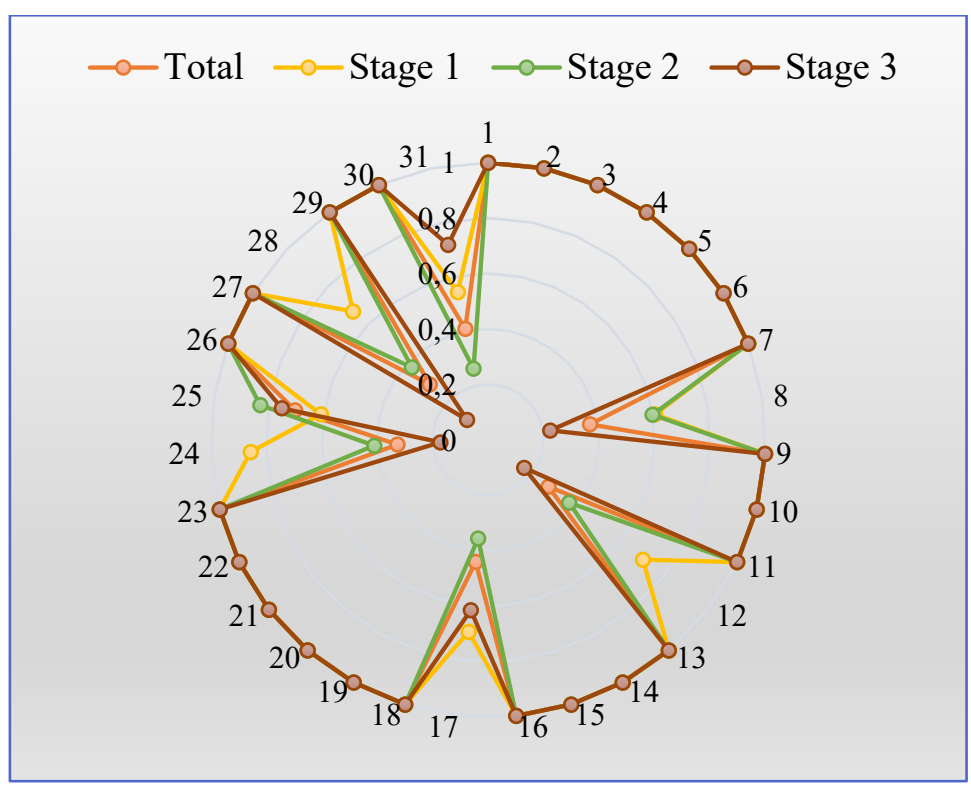

Fig. 3. Overall and subsections efficiency scores

The second column in Table 2 shows the whole system's overall efficiency. Columns 3-5 show the efficiency score of each stage in the 31 regions. Given the values of these columns, the result is obtained that units are overall efficient if and only if every stage is efficient. In fact, inefficiency of each stage leads to overall inefficiency. This case is also observed in Fig. 3, when some lines are tangent.

The number of efficient units in columns $2-5$ shows that, in whole system and at every stage, $22.59 \%$ of the units are inefficient and the rest are efficient. According to columns $3-5$, it can be found that efficiency of some regions in stage 1 is the less than stage 2 and 3, and for some it is different. For example, the measure efficiency of region 12 in stage 3 is the less than its other stages, or the measure efficiency of region 31 in stage 2 is the less than it in other stages. In general, columns 3-5 show that the most inefficient units in the stages 1,2 and 3 are 31,31 , and 28 , respectively.

Columns 6 and 7 show the optimal portion value for the shared input of stage 1 and 2 . For example, unit 1 needs $\alpha^{(1)^{*}}=0.3092$ portion from $x^{(1)}$ and $\alpha^{(2)^{*}}=0.6908$ portion from $x^{(2)}$, when optimality is considered. Optimal portions for the stage 1 and stage 2 are not the same. In other words, the optimal portion of some regions for stage 1 is more than stage 2 and for some others it is the reverse. For instance, the optimal portion of region 26 for stage 1 is 0.5094 , while this portion for stage 2 is 0.4906 . In the other cases, like in region 19 , the optimal portion for stage 1 is 0.3133 , which is lower than the optimal portion of stage 2 with the amount of 0.6867 . 
Table 2. Results of models (4) and (9)

\begin{tabular}{|c|c|c|c|c|c|c|c|}
\hline Region & $e_{o}^{* \text { Overall }}$ & $e_{o}^{* \text { Stage } 1}$ & $e_{o}^{* \text { Stage } 2}$ & $e_{o}^{* \text { Stage } 3}$ & $\alpha^{(1)^{*}}$ & $\alpha^{(2)^{*}}$ & $\sigma_{o}^{* \text { Overall }}$ \\
\hline 1 & 1.0000 & 1.0000 & 1.0000 & 1.0000 & 0.3092 & 0.6908 & $3.4259(2)$ \\
\hline 2 & 1.0000 & 1.0000 & 1.0000 & 1.0000 & 0.1677 & 0.8323 & $2.1888(11)$ \\
\hline 3 & 1.0000 & 1.0000 & 1.0000 & 1.0000 & 0.4112 & 0.5888 & $2.0735(15)$ \\
\hline 4 & 1.0000 & 1.0000 & 1.0000 & 1.0000 & 0.4029 & 0.5971 & $2.2410(10)$ \\
\hline 5 & 1.0000 & 1.0000 & 1.0000 & 1.0000 & 0.4279 & 0.5721 & $2.5886(6)$ \\
\hline 6 & 1.0000 & 1.0000 & 1.0000 & 1.0000 & 0.3699 & 0.6301 & $2.8881(5)$ \\
\hline 7 & 1.0000 & 1.0000 & 1.0000 & 1.0000 & 0.3202 & 0.6798 & $3.2251(4)$ \\
\hline 8 & 0.3711 & 0.6088 & 0.5998 & 0.2265 & 0.2465 & 0.7535 & - \\
\hline 9 & 1.0000 & 1.0000 & 1.0000 & 1.0000 & 0.3148 & 0.6852 & $2.0856(14)$ \\
\hline 10 & 1.0000 & 1.0000 & 1.0000 & 1.0000 & 0.2900 & 0.7100 & $2.1540(13)$ \\
\hline 11 & 1.0000 & 1.0000 & 1.0000 & 1.0000 & 0.2182 & 0.7818 & $2.4067(8)$ \\
\hline 12 & 0.2751 & 0.7055 & 0.3697 & 0.1639 & 0.1302 & 0.8698 & - \\
\hline 13 & 1.0000 & 1.0000 & 1.0000 & 1.0000 & 0.2417 & 0.7583 & $2.1840(12)$ \\
\hline 14 & 1.0000 & 1.0000 & 1.0000 & 1.0000 & 0.2833 & 0.7167 & $1.8406(22)$ \\
\hline 15 & 1.0000 & 1.0000 & 1.0000 & 1.0000 & 0.4579 & 0.5421 & $3.2719(3)$ \\
\hline 16 & 1.0000 & 1.0000 & 1.0000 & 1.0000 & 0.3189 & 0.6811 & $1.9799(18)$ \\
\hline 17 & 0.4419 & 0.6955 & 0.3574 & 0.6171 & 0.1458 & 0.8542 & - \\
\hline 18 & 1.0000 & 1.0000 & 1.0000 & 1.0000 & 0.1736 & 0.8264 & $1.8685(20)$ \\
\hline 19 & 1.0000 & 1.0000 & 1.0000 & 1.0000 & 0.3133 & 0.6867 & $2.3299(9)$ \\
\hline 20 & 1.0000 & 1.0000 & 1.0000 & 1.0000 & 0.3113 & 0.6887 & $1.8684(21)$ \\
\hline 21 & 1.0000 & 1.0000 & 1.0000 & 1.0000 & 0.2187 & 0.7813 & $2.5565(7)$ \\
\hline 22 & 1.0000 & 1.0000 & 1.0000 & 1.0000 & 0.1446 & 0.8554 & $1.6713(24)$ \\
\hline 23 & 1.0000 & 1.0000 & 1.0000 & 1.0000 & 0.3084 & 0.6916 & $1.7936(23)$ \\
\hline 24 & 0.3277 & 0.8576 & 0.4114 & 0.1726 & 0.0829 & 0.9171 & - \\
\hline 25 & 0.7043 & 0.6103 & 0.8318 & 0.7531 & 0.2769 & 0.7231 & - \\
\hline 26 & 1.0000 & 1.0000 & 1.0000 & 1.0000 & 0.5094 & 0.4906 & $63.4737(1)$ \\
\hline 27 & 1.0000 & 1.0000 & 1.0000 & 1.0000 & 0.2373 & 0.7627 & $1.9529(19)$ \\
\hline 28 & 0.2907 & 0.6724 & 0.3796 & 0.1051 & 0.1599 & 0.8401 & - \\
\hline 29 & 1.0000 & 1.0000 & 1.0000 & 1.0000 & 0.5750 & 0.4250 & $2.0231(17)$ \\
\hline 30 & 1.0000 & 1.0000 & 1.0000 & 1.0000 & 0.6461 & 0.3539 & $2.0668(16)$ \\
\hline 31 & 0.4091 & 0.5444 & 0.2624 & 0.7183 & 0.1469 & 0.8531 & - \\
\hline & & & & & & & \\
\hline 13 &
\end{tabular}

As can be seen in column 2, 24 regions out of 31 ones had a desirable performance. Hence, to analyse the performance of efficient units and to distinguish between them, we applied model (9) to the mentioned example. The results obtained from ranking efficient units for the whole system in this way are significant in the last column. Column 8 shows that region 26 has the first rank, and it means that the mentioned unit has a better performance than the other efficient units. Also, region 22 has the 24th rank or the last rank, and it means that the mentioned unit does not have good performance among all the 24 efficient units. 


\section{Conclusions}

In the real world, there are systems with complex structures that include processes, whose patterns are like parallel-series mixed network. The presence of undesirable factors in the structure of such processes makes them more complex. The existing studies introduce different models for the mixed network process. But, as far as we know, none of these models uses weak disposability assumption to handle undesirable outputs.

Accordingly, in this paper, we consider $D M U$ s with the parallel-series mixed network structure, in which stages 1 and 2, in addition to their inputs, consume the shared inputs and produce desirable and undesirable outputs. Stage 3 utilises the inputs from the previous stages in addition to the extra inputs and produces desirable and undesirable outputs. Also, the weak disposability assumption for undesirable and desirable factors is considered, which makes the provided approach fundamentally different from the previous studies.

We present a model built on the SBM model for assessing the relative efficiency of units with the parallel-series mixed structure. The introduced model can calculate the overall relative efficiency without the need to evaluate the efficiency of each of the subsections. Also, it can evaluate the efficiency of each of the subsections for the mentioned mixed structure. Besides, the proposed method determines an optimal portion amount of the shared inputs for the parallel section.

Given that sometimes it is important to know which unit is the most efficient one among the other efficient units, a super-efficiency model is introduced to determine the rank of those units. For further analysis, the introduced model was applied to evaluate the performance of whole systems and their sub-sections of 31 regions of China. The findings show that the suggested approach can assess the efficiency of mixed network processes with undesirable outputs and rationally shared inputs.

Estimating the performance of different network processes with various stages, including non-controllable and undesirable variables, can be an extension of the proposed approach and is an interesting topic for future investigation.

\section{References}

[1] Amirteimoori A., Kordrostami S., Azizi H., Additive models for network data envelopment analysis in the presence of shared resources, Transp. Res. D., 2016, 48, 411-424.

[2] AMIRTEIMOORI A., YANG F., A DEA model for two-stage parallel-series production processes, RAIRO Oper. Res., 2014, 48, 123-134.

[3] BIAN Y., YAN S., XU H., Efficiency evaluation for regional urban water use and wastewater decontamination systems in China. A DEA approach, Res. Cons. Rec., 2014, 83, 15-23.

[4] Charnes A., CoOper W.W., Rhodes E., Measuring the efficiency of decision-making units, Eur. J. Oper. Res., 1978, 2, 429-444. 
[5] CHEN Y., CoOK W.D., Li N., ZHU J., Additive efficiency decomposition in two-stage DEA, Eur. J. Res., 2009, 196, 1170-1176.

[6] Chen Y., Du J., SHERMAn H.D., ZHu J., DEA model with shared resources and efficiency decomposition, Eur. J. Oper. Res., 2010, 207, 339-349.

[7] CHEN Y., ZHU J., Measuring information technology's indirect impact on firm performance, Inf. Technol. Manage., 2004, 5, 9-22.

[8] Färe R., Grosskopf S., Network DEA, Socio-Econ. Plan. Sci., 2000, 34, 35-49.

[9] FÄre R., GrossKopf S., Nonparametric productivity analysis with undesirable outputs. Comment, Am. J. Agr. Econ., 2003, 85, 1070-1074.

[10] KAO C., Efficiency measurement for parallel production systems, Eur. J. Oper. Res., 2009, 196, 1107-1112.

[11] KaO C., Hwang S.-N., Efficiency decomposition in two-stage data envelopment analysis: An application to non-life insurance companies in Taiwan, Eur. J. Oper. Res., 2008, 185, 418-429.

[12] KuOSMANEN T., Weak disposability in nonparametric production analysis with undesirable outputs, Am. J. Agr. Econ., 2005, 87, 1077-1082.

[13] Liang L., COOK W.D., ZHU J., DEA models for two-stage processes. Game approach and efficiency decomposition, Nav. Res. Log., 2008, 55, 643-653.

[14] LIN T.Y., CHIU S.H., Using independent component analysis and network DEA to improve bank performance evaluation, Econ. Model., 2013, 32, 608-616.

[15] MA J., CHEN L., Evaluating operation and coordination efficiencies of parallel-series two-stage system: A data envelopment analysis approach, Exp. Syst. Appl., 2018, 91, 1-11.

[16] Maghbouli M., Amirteimoori A., Kordrostami S., Two-stage network structures with undesirable outputs. A DEA based approach, Measurement, 2014, 48, 109-118.

[17] Nematizadeh M., Amirteimoori A., Kordrostami S., Performance analysis of two-stage network processes with feedback flows and undesirable factors, Oper. Res. Dec., 2019, 3, 51-66.

[18] SeIford L.M., Zhu J., Profitability and marketability of the top 55 US commercial banks, Manage. Sci., 1999, 45, 1270-1288.

[19] ShePhard R.W., Theory of Cost and Production Functions, Princeton University Press, Princeton 1970.

[20] TONE K., A slack-based measure of super-efficiency in data envelopment analysis, Eur. J. Oper. Res., 2002, 143, 32-41.

[21] Tone K., TsuTsui M., Network DEA. A slack-based measure approach, Eur. J. Oper. Res., 2009, 197, $243-252$.

[22] Wu J., Zhu Q., CHU J., LiU H., LiAnG L., Measuring energy and environmental efficiency of transportation systems in China based on a parallel DEA approach, Trans. Res. D., 2016, 48, 460-472.

[23] YU M.M., Assessment of airport performance using the SBM-NDEA model, Omega, 2010, 38, 440-452.

[24] YU M.M., LIN E.T.J., Efficiency and effectiveness in railway performance using a multi-activity network DEA model, Omega, 2009, 36, 1005-1017.

[25] Zha Y., Liang L., Two-stage cooperation model with input freely distributed among the stages, Eur. J. Oper. Res., 2010, 205, 332-338.

[26] ZhaO L., Sun C., Liu F., Interprovincial two-stage water resource utilization efficiency under environmental constraint and spatial spillover effects in China, J. Clean. Prod., 2017, 164, 715-725. 